\section{Schützt potenziertes Glutamat vor Neurotoxizität?}

\section{Jonas W Lin Y, Tortella F: Neuroprotection from glutamate toxicity with ultra-low dose glutamate. NeuroReport 2001;12:335-339.}

Background: Glutamate is a neurotoxic substance which is held responsible for some of the damage observed in stroke. In an animal stroke model (primary rat spinal, cortical and cerebellar neurons) the protective effects of ultra-low doses (ULD) of glutamate against glutamate toxicity were studied. Method: Neurons were exposed to four subtoxic, ultra-low concentrations of glutamate $\left(10^{-18} \mathrm{M}, 10^{-20} \mathrm{M}, 10^{-22} \mathrm{M}\right.$ and $10^{-30} M$; i.e. homeopathic potencies of $\mathrm{C} 18, \mathrm{C} 20, \mathrm{C} 22$ and $\left.\mathrm{C} 30\right)$ for $72 \mathrm{~h}$ and then subsequently challenged with toxic concentrations $(25 \mu M)$ of glutamate.

Results: Neuron viability was consistently $10 \%$ higher in spinal and cortical neurons pre-exposed to glutamate concentrations of $10^{-18} M$ and $10^{-22} M$, and in cerebellar neurons pre-exposed to $10^{-20} M$ and $10^{-30} M$. Using laser scanning confocal microscopy and the fluorescent calcium probe fluo-3, we found no alterations in intracellular calcium dynamics in the protected cells. This protective effect is consistent with a growing body of evidence for tolerance induced by low-dose toxin exposure but is the first time that such tolerance has been demonstrated with ultra-low glutamate exposure.

Conclusion: Our data show that pre-exposure of neuronal cells to ULD glutamate can protect against subsequent exposure to toxic levels of glutamate.

\section{Kommentar - R. van Wijk, Utrecht}

The reviews and meta-analyses examining whether a high potency homeopathic remedy has any greater effect than placebo have raised the question of developing high-quality studies based on randomization, reproducibility and standardizability, allowing structured statistical analysis. However, the latter is seldom possible in clinical trials. It has led to the search for reliable, nonhuman model systems that are more simple to handle and allow such critical studies.

Jonas et al. explored the phenomenon of neurotoxicity of glutamate and studied in detail the protection by pre-exposure to ultra-low concentrations. In their study they used primary cultures from forebrain, cerebellum and spinal cord of prenatal rats. The endpoint for neurotoxicity is the simple and straight forward parameter of viability of cells. The procedure of viability assessment includes the tetrazolium salt colorimetric assay. In the present studies this assay was carried out 18-20 h after intoxication by a toxic dose of glutamate (30 $\mathrm{min}, 25$ $\mu M)$. In the study the protective effect of four ultra-low concentrations of glutamate were studied. The four preparations were made by serial (1:100) dilutions of vigorously mixed dilutions and were indicated as $10^{-18}, 10^{-20}, 10^{-22}$, and $10^{-30} \mathrm{M}$.

With respect to methodological aspects of the experiment, cells were cultured in multi-well plates with pre-exposure and control conditions in the same plate, and more plates each experimental day. Most interesting is the outcome after combining the experimental data obtained at different experimental days with cells showing roughly the same sensitivity for glutamate intoxication, namely $70 \%$ viability after treatment with $25 \mu M$ glutamate without any pretreatment. This resulted in a reasonable number of samples per pre-exposure condition for spinal neurons $(n=60)$, cortical neurons $(n=72)$, and cerebellar neurons $(\mathrm{n}=33)$.

The data of each pre-exposure condition were compared with the non-pre-exposure condition. Some pre-exposure conditions showed a protective effects of $10 \%$ more viable cells. The pre-exposure conditions resulting in this protection depended on cell type. Protection at statistical significant level was observed in cortical neurons at $10^{-18} \mathrm{M}$, in spinal neurons at $10^{-18}$ and $10^{-22} \mathrm{M}$, and in cerebellar neurons at $10^{-20}$ and $10^{-30} \mathrm{M}$.

At first glance these results are straight away convincing and in line with earlier studies of Delbancut et al. [1] on renal cells pre-exposed to ultra-low levels of cadmium.

However, the term cell viability, expressed as \% of control value, requires some additional explanation. In fact, an interesting feature of a simple biological model system is that it can be used for searching into the biological mechanism explaining this $10 \%$ extra cell viability. In the present study this difference in cell viability is expressed without taking into account any difference in the absolute number of cells between the control and pre-exposed conditions. Since pre-exposure time was $72 \mathrm{~h}$, this time period is enough to allow an effect on absolute cell number by influences on growth rate or rate of cell death.

However, this does not alter the conclusion that this type of protocol needs again our attention and at least a careful repetition by another experienced laboratory.

\section{Reference}

1 Delbancut A, Barouillet MP, Cambar J: Evidence and mechanistic approach of the protective effects of heavy metal high dilutions in rodent and in renal cell cultures; in Bashide M (ed): Signals and Images. Dordrecht, Kluwer, 1997, pp 71-82.

Kontaktadresse: Dr. Roeland Van Wijk, Koppelsedijk 1A, NL-4191 LC Geldermalsen, E-mail meluna.wijk@wxs.nI

Adresse für Sonderdrucke: Dr. Wayne Jonas, Department of Family Medicine, Program in Neuroprotection, Uniformed Services University of the Health Sciences, 4301 Jones Bridge Road, Bethesda, MD 20814, USA, EmailWBJonas@aol.com 